\title{
Simulation Based Investigation of Hidden Delamination Damage Detection in CFRP Composites
}

\author{
Cara A.C. Leckey and F. Raymond Parker \\ Nondestructive Evaluation Sciences Branch, NASA Langley Research Center, Hampton VA 23681
}

\begin{abstract}
Guided wave (GW) based damage detection methods have shown promise in structural health monitoring (SHM) and hybrid SHM-nondestructive evaluation (NDE) techniques. Much previous GW work in the aerospace field has been primarily focused on metallic materials, with a growing focus on composite materials. The work presented in this paper demonstrates how realistic three-dimensional (3D) GW simulations can aid in the development of GW based damage characterization techniques for aerospace composites. $3 \mathrm{D}$ elastodynamic finite integration technique is implemented to model GW interaction with realistic delamination damage. A local wavenumber technique is applied to simulation data in order to investigate the detectability of hidden delamination damage to enable accurate characterization of damage extent.
\end{abstract}

Keywords: Composite, guided wave, delamination, simulation, EFIT

PACS: 43.35.Zc, 43.20.Gp, 43.35.Cg

\section{INTRODUCTION}

Guided wave (GW) based methods have shown promise in structural health monitoring (SHM) and hybrid SHMnondestructive evaluation (NDE) damage detection techniques. Much previous GW work for aerospace applications has been primarily focused on metallic materials, with a growing focus on composite materials. One example of a hybrid SHM-NDE damage detection technique is the use of a sparse piezoelectric sensor array for determining damage location (or a region of interest that may contain damage), followed by use of a scanning laser Doppler vibrometer (LDV) for wavefield data capture, as depicted in the diagram in figure 1. The in-situ piezoelectric sensors send and receive in a round-robin setup, collecting GW data that is processed to create damage maps depicting damage location [1]. The same sensors are then excited to create a GW wavefield that is measured by the LDV as it interacts with any damage in the region of interest. A variety of LDV wavefield data processing techniques have been explored in the literature for the purpose of damage quantification and characterization. Recent examples include the use of incident wave removal via wavenumber domain filtering and local wavenumber domain analysis, among other methods [2-3]. Scanning air-coupled ultrasonic techniques have also been used for wavefield data capture and could similarly be combined with an SHM technique for a hybrid approach [4].

Most prior GW simulation studies on damage detection techniques for composites make simplifying assumptions about damage geometry (for example, including a single planar or circular delamination) [5]. The work presented in this paper is intended to demonstrate that more realistic three-dimensional (3D) GW simulations should be used to aid the development of GW based damage characterization techniques for aerospace composites. Simulations that account for GW interaction with the complex geometries that exist for real delamination damage may help researchers leverage unique wave behavior in order to extract additional information about the damage that is present.

The 3D elastodynamic finite integration technique (EFIT) is implemented to model GW interaction with realistic delamination damage. Previous work by the authors reports details of the anisotropic EFIT approach as well as prior work on validation of the 3D anisotropic EFIT code [6]. 3D EFIT simulations can output the out-of-plane velocity wavefield data at the composite sample surface, analogous to the velocity wavefield data that can be collected experimentally using a scanning one-dimensional (1D) LDV. In addition to surface wavefield information for a composite sample, the simulations can also output both out-of-plane and in-plane velocity for all depths through the composite thickness. In this paper we demonstrate the use of local wavenumber domain analysis applied to simulated wavefield data at the composite laminate surface to investigate the detectability of hidden delamination damage in order to enable accurate characterization of damage extent. 


\section{SIMULATION OF GW INTERACTION WITH DELAMINATION DAMAGE}

The custom EFIT code models each ply layer of the composite laminate sample. This level of detail is required in order to capture the ply-direction-dependent geometric complexity of real delaminations and the related GW behavior. In order to acquire realistic delamination data to incorporate into the EFIT GW simulations, a quasi-static indentation technique was used for controlled growth of impact-like delamination damage in a 26 ply thick quasiisotropic IM7/8552 composite laminate sample with layup $\left[(0 /+45 /-45 / 90)_{3}\right]_{\mathrm{s}}$. Both ultrasonic C-scan data and Xray computed tomography $(\mathrm{CT})$ data were taken of the resulting delamination damage.

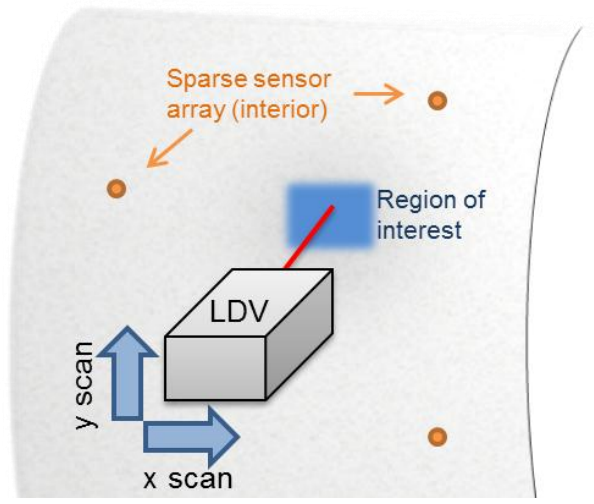

FIGURE 1. Diagram showing one concept for a hybrid NDE-SHM method: An in-situ sparse piezoelectric sensor array is used to locate suspected damage regions via guided wave techniques. Once a region of interest is identified, an NDE technique, such as wavefield data capture using a scanning LDV, is used to provide additional quantitative damage characterization information.

Figure 2a shows individual image slices taken from the volumetric X-ray CT data. Figures $2 \mathrm{~b}$ and $2 \mathrm{c}$ show the delamination data that was pulled from the X-ray CT slices and read directly into the EFIT simulations. Stress-free boundaries were implemented in the EFIT simulation at grid cells corresponding to damage voxels. The EFIT simulations discussed in this paper used a spatial step size of 23.4 microns, matching the resolution of the X-ray CT data in order to capture the details of the delamination damage geometry. Prior work by the authors further discusses the incorporation of X-ray CT damage maps into EFIT simulations [6]. As shown in figure 2b, the delaminations spiral downward through the laminate thickness, alternating lateral growth directions according to changes in ply direction. Figure 3a shows data from a single-sided ultrasonic C-scan (time of flight data) of the composite sample and was taken from the impacted side of the sample. Note that the image is rotated counter clockwise from the image in figure $2 \mathrm{c}$ and uses a different colorscale. Figure $3 \mathrm{~b}$ shows a 'hidden' delamination, circled by the dashed red line, occurring towards the back surface of the sample (the surface opposite of the impact). This backmost delamination is not visible in the single sided ultrasonic $\mathrm{C}$-scan, and would likely also not be detectable with other traditional single-sided NDE techniques. For aerospace applications, the assumption of singlesided access (e.g., the outside of a vehicle) is generally a reasonable real-world assumption.

The presence of hidden delamination damage, which has also been observed in X-ray CT data from other impacted composite laminate samples at NASA Langley, led us to explore the use of realistic 3D GW simulations for investigating potential damage characterization techniques for damage scenarios such as this. Prior work by Canturri et. al., among others, has shown that the extent of damage through the laminate thickness (i.e., the location of delaminations at various ply depths through the thickness) is important for accurate damage growth predictions, and thus for remaining life predictions for composite components [7]. Three simulation cases were implemented using the custom EFIT code in order to explore differences in GW interaction with varying amounts of delamination damage extending through the composite laminate thickness. The three damage cases are shown in figure 3c: 1) the full delamination is included; 2) all damage from the hidden delamination depth downward is removed and replaced with pristine material (i.e., there is no damage below the dashed plane intersecting the damage shown in figure $3 \mathrm{c} ; 3$ ) half of the delamination damage (by depth) has been removed and replaced with pristine material. A $400 \mathrm{kHz} 5$-cycle Hann windowed sine wave was excited over a 0.5 inch circular region on the simulated composite sample. The small EFIT simulation step size (23.4 microns) leads to a total simulation size over 1.8 billion grid cells. Each simulation case was run on an 80 core 1TB memory HP Proliant DL-980 system and took approximately two weeks of run time. 


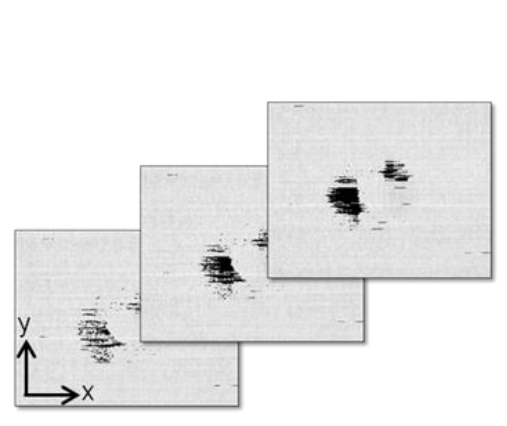

(a)

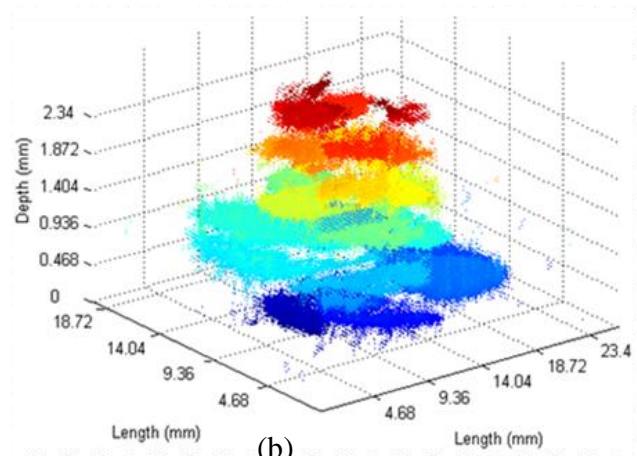

(b)

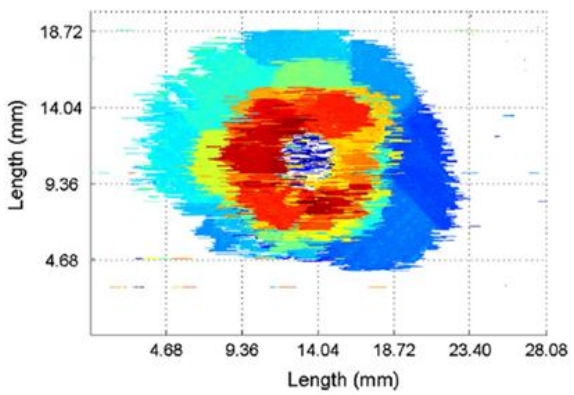

(c)

FIGURE 2. (a) Image slices from X-ray CT data taken of the delaminated composite laminate sample, (b) Volumetric delamination data created from X-ray CT slices and incorporated directly into the EFIT simulation. The colorscale corresponds to depth on the $\mathrm{z}$-axis. The impact occurred at the sample surface corresponding to depth $\mathrm{z}=3.23 \mathrm{~mm}$, (c) Top view of the $\mathrm{X}$-ray CT delamination data (viewed from impact side).

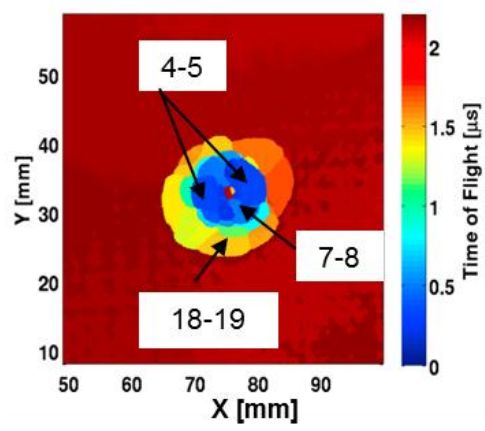

(a)

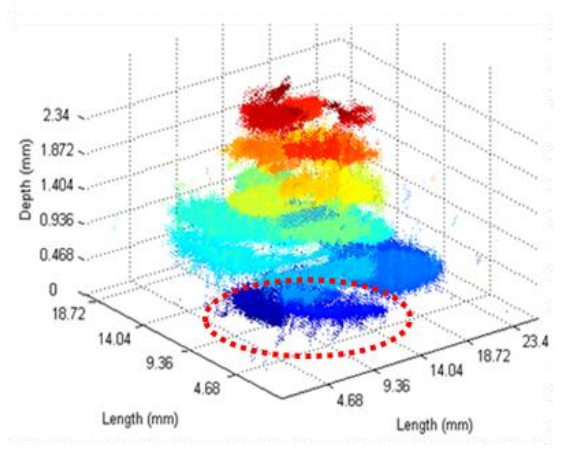

(b)

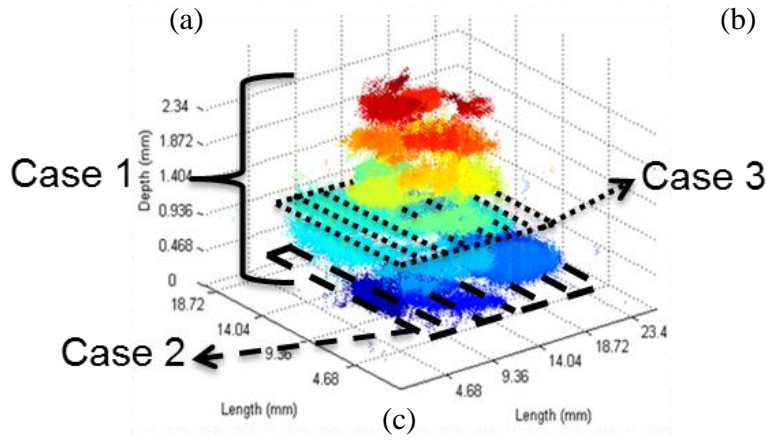

FIGURE 3. (a) Single-sided C-scan data of the delamination damage, with white boxes listing ply depths between which some of the delamination damage occurs (identified through time of flight). The figure is taken from reference [3], (b) X-ray CT data with the 'hidden' delamination region circled in red, (c) three EFIT simulation cases that were implemented for this paper, Case 1: the full delamination damage is included in the simulation, Case 2: hidden delamination damage is removed and replaced with pristine material below the lower dashed plane intersecting the image, Case 3: half of the delamination damage is removed. 


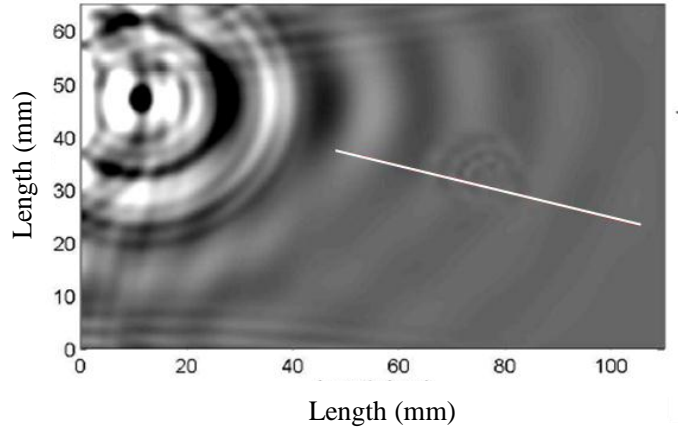

(a)

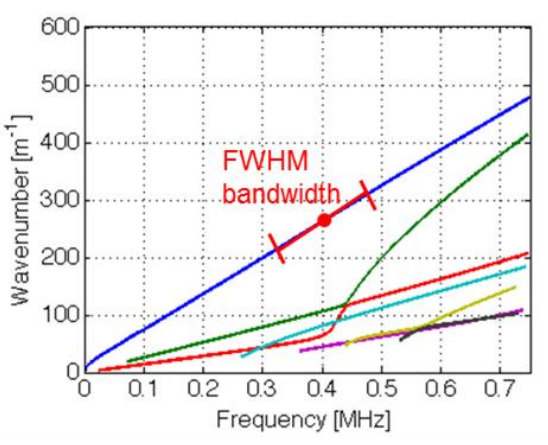

(b)

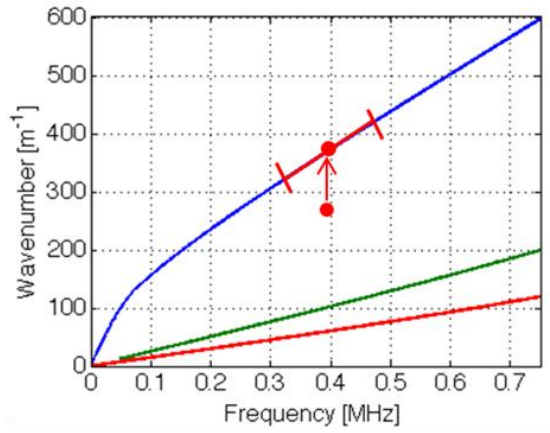

(c)

FIGURE 4. (a) Snapshot of the simulated wavefield (out-of-plane velocity) at the composite surface, showing an S0-type GW mode interacting with the full delamination damage (case 1), (b) dispersion curves for the full composite plate thickness with the bandwidth of the excitation shown in red, (c) dispersion curves for the top four plies of the composite sample, showing the expected shift in wavenumber above the uppermost delamination damage (indicated by the red dot and arrow shifting upward from the wavenumber of 26 ply curve).

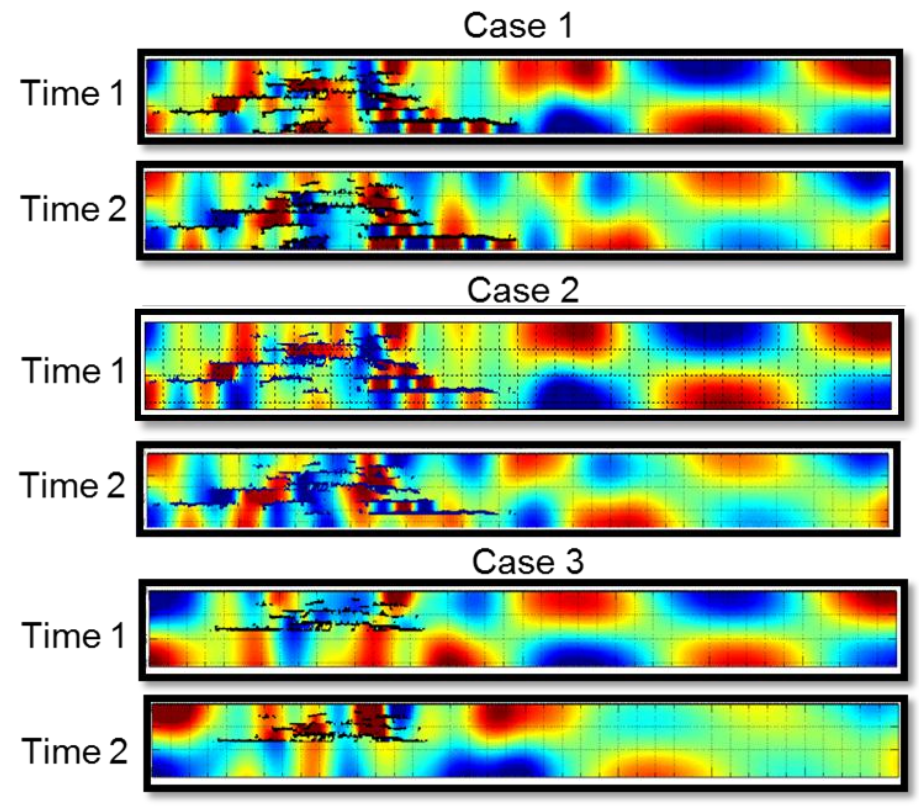

FIGURE 5. Thickness slices taken from the 3D EFIT simulations along the position of the solid white line in figure 4a. The images show the out-of-plane velocity through the laminate thickness at two different points in time after the initial excitation for each of the three cases.

A single time snapshot of the simulated wavefield at the laminate surface is shown in figure 4a (excitation located in the upper left corner). The full delamination damage is centered at approximately $76 \mathrm{~mm}$ on the $\mathrm{x}$-axis and $35 \mathrm{~mm}$ on the $\mathrm{y}$-axis. Figure $4 \mathrm{~b}$ shows dispersion curves for the full composite sample thickness and $4 \mathrm{c}$ shows curves for the top four plies of the laminate. The dispersion curves were created using DISPERSE software [8]. These curves demonstrate the shift in wavenumber that occurs as waves enter into 'thinned' regions above (or between) delaminations. Changes in group velocity can also occur as waves enter regions of various thicknesses.

Figure 5 shows thickness slices taken from the 3D EFIT simulations along the position of the solid white line in figure $4 \mathrm{a}$ at two different snapshots in time: time 1 is $20.47 \mu$ s after the initial excitation and time 2 is $22.00 \mu$ after the excitation. The colormap represents the out-of-plane velocity with red representing large positive values, and blue representing large negative values. An incident S0-type mode is moving from left to right in the images shown in figure 5. It is clearly visible in the figure that complex changes in the GW modes occur above and between delaminations in the damage region (located in the left half of the images, with black lines indicating delaminations). 
Shifts in wavenumber can be observed as changes in wavelength of the modes, compared to the longer wavelength observed in the full plate thickness to the right of the damage (particularly visible at time 1 before significant scattered waves emerge from the damage region). Additionally, at time 1 it can be observed (at the far right of the time 1 images in figure 5) that the differences in mode conversion between the three cases lead to very little change in travel time for the re-emerged full-plate-thickness S0-type mode. At time 2 scattering can be observed to the right of the damage. Differences in the scattered field can be observed for the three cases. Yet, based on the simulation results, it appears that using a sparse array GW SHM system alone is unlikely to result in arrival time based damage maps that differentiate between the severity levels of damage in the three cases. As mentioned earlier, if a GW SHM system can at least identify a region of interest, an NDE technique such as LDV wavefield measurements could then be used to further characterize any damage that exists within the region of interest.

\section{APPLICATION OF LOCAL WAVENUMBER ANALYSIS}

If wavefield data for a region of interest is available via LDV measurements, then wavefield analysis techniques can be used to quantitatively determine damage size and the depth of near-surface (non-hidden) delaminations. Prior work by Rogge et. al. showed that local wavenumber domain analysis technique can be used to create a 2D space-wavenumber map of the damage region [3]. In this method, a 3D Fourier transform (FFT) is applied to spatially windowed regions of the wavefield data to yield information about the dominant wavenumber versus $\mathrm{x}$ and y position. The process is accomplished as shown in figure 6. First, a 2D Hann window is applied to select a local spatial region of the wavefield for all points in time (6a). Next a 3D FFT is taken of the windowed space-time domain data to yield wavenumber-frequency domain data (6b). The frequency slice corresponding to the center excitation frequency is then chosen (6c) and the dominant wavenumber is calculated using a weighted mean (6d). The resulting wavenumber is plotted for the position corresponding to the center of the window location, the next window position is chosen and the process repeated to build up a 2D space-wavenumber plot (neighboring spatial window positions can be chosen such that windows overlap).

Rogge et. al. showed that the spatial window size should be at least twice the guided wave mode wavelength to accurately resolve the local wavenumber [3]. In practice, for experimental LDV wavefield data of a damage region, the expected wavenumbers for possible damage scenarios/depths may be unknown (especially if material properties or layup are unknown). For this case a sweep in window size may be required. As the window size reaches the ideal size (window $\geq 2 \lambda$ ) and increases further, the predicted wavenumber will converge towards the actual value. However, as the window size is increased, the ability to resolve spatial changes in wavenumber decreases since waves over a larger region contribute towards the calculated dominant wavenumber.

Similar to the traditional one-sided NDE methods, such as the ultrasonic data shown in figure 3a, this technique would also not readily resolve the type of hidden damage observed in the x-ray CT data. However, if the technique is also applied to regions beyond the damage, wavenumber differences in the scattered field may lead to readily observable differentiating evidence between various damage scenarios, such as the three that are investigated here. To explore this possibility, the local wavenumber domain analysis technique was applied to simulated wavefield data for the three different damage cases. In order to decrease processing time of the 3D FFT, only a portion of the total simulated out-of-plane wavefield data from the composite laminate surface was chosen for further processing (as shown in figure 6a, compared to figure 4a). A local window size of $5.62 \mathrm{~mm}$ was used for the results presented in this paper. This window size corresponds to approximately $2 \lambda$ for the expected wavelength of the S0-type mode above the uppermost delamination (located 4 plies below the top surface), and is based on the dispersion curves shown in figure $4 \mathrm{c}$. The results are shown in figure 7 , where the colormap represents wavenumber and the $\mathrm{x}$ and $\mathrm{y}$ axes are position in $\mathrm{mm}$. The first observation about the local wavenumber images is that the unhidden delamination damage has been sized fairly accurately. For case 1 the wavenumber plot shows a delamination area of approximately $18.3 \mathrm{~mm}$ by $14.2 \mathrm{~mm}$, while the x-ray CT data in figure 2c (also for case 1) shows a delamination area of around $18.7 \mathrm{~mm}$ by $14.7 \mathrm{~mm}$. The decrease in delamination area for the three different cases is apparent in the local wavenumber images. In fact, the full extent of damage area reduction for case 2 was unclear before the local wavenumber results were observed. It was expected that removing delamination damage from the selected zaxis depth downward would not affect the damage geometry as viewed from the impacted surface. However, it is clear from the results in figure $7 \mathrm{~b}$ that the hidden delamination extends upward slightly into a depth where damage voxels of the larger delamination also exist. The unintended removal of small portions of the larger 'non-hidden' delamination in case 2 can also be observed through careful re-examination of the deepest delamination in the EFIT thickness plots in figure 5, but is not as apparent as the wavenumber results. 


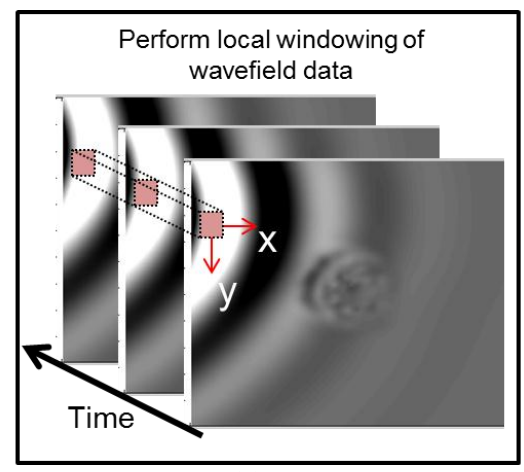

(a)

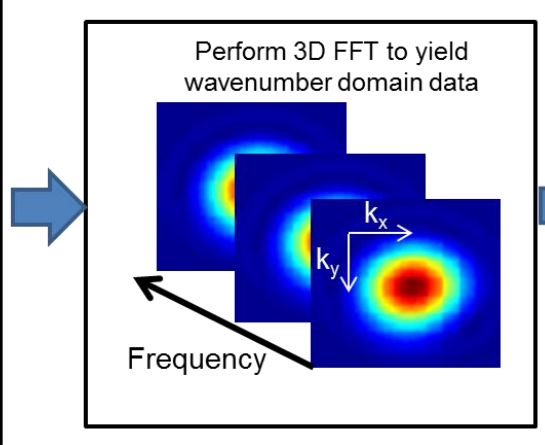

(b)

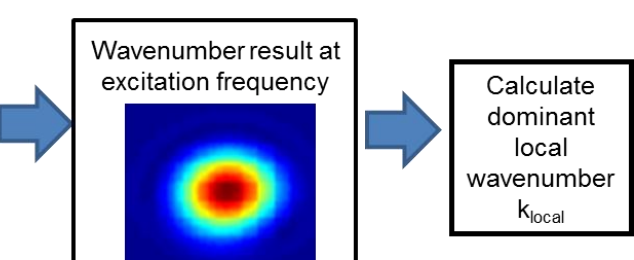

(c) (d)

FIGURE 6. Diagram showing the local wavenumber domain calculation process: (a) Select local spatial window, (b) calculate 3D FFT of local space-time data to yield wavenumber-frequency data, (c) select excitation frequency, (d) calculate dominant local wavenumber.

The wavenumber values in figure 7 can be directly correlated to delamination depth via dispersion curves. For example, the uppermost delamination damage corresponds to the largest wavenumber (red on the colorscale) since the corresponding dispersion curve shifts upward with decreasing thickness (as shown in figure 4c). The local wavenumber analysis predicts an average wavenumber around $360 \mathrm{~m}^{-1}$ in the regions corresponding to the upper delamination damage. This value correlates well with the dispersion curve prediction of approximately $368 \mathrm{~m}^{-1}$ for damage located four plies beneath the surface.

Another observation that can be made regarding the results in figure 7 is that the differences in the scattered field that were noted in the discussion of figure 5 are also apparent in the local wavenumber domain images. In order to place a specific quantitative value on the scattered field wavenumber differences between the three cases, the local wavenumber domain results for cases 2 and 3 were subtracted from the results for case 1 to yield differenced local wavenumber images (figure 8). The significant wavenumber changes due to differences in overall damage geometry are immediately apparent in the damage region, and show up as large differences in wavenumber (maxing out the color scale in figure 8). Note that the colormap used in figure 8 is slightly saturated in order to make scattered field wavenumber changes more apparent. To the right of the damage region, a wavenumber difference around $15 \mathrm{~m}^{-1}$ can be observed for case 2 minus case 1 . A slightly larger difference, around $25 \mathrm{~m}^{-1}$, is observed in the scattered field for case 3 minus case 1 .

Although the results in figures 7 and 8 demonstrate how local wavenumber domain analysis can be applied to wavefield data to readily determine quantitative differences in the scattered field between the three damage cases, significant additional research is required to make more definite and specific connections between the presence of 'hidden' damage and wavenumber domain results. A simulation including damage data after a more precise removal of the 'hidden' damage will be performed in the future in order to leave the in-plane damage extent unaffected and allow for a more isolated study of GW behavior due to hidden through-thickness damage.

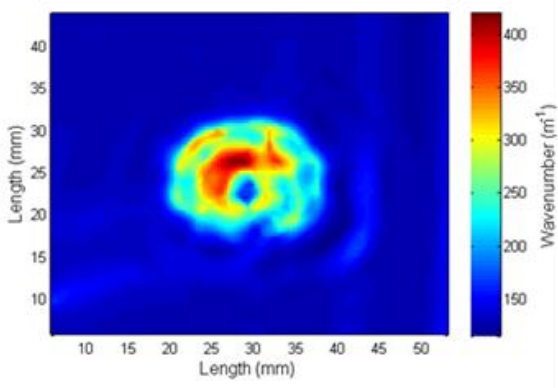

(a)

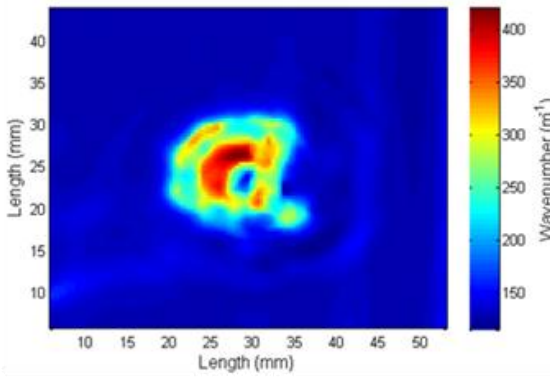

(b)

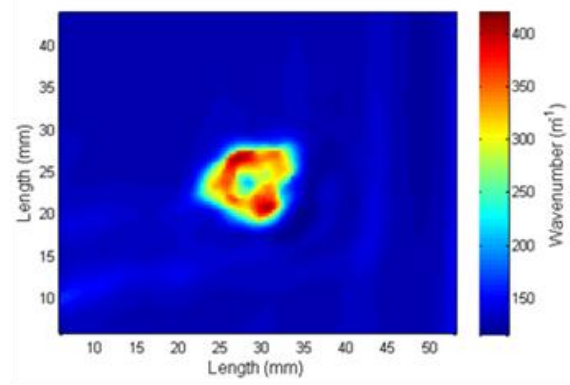

(c)

FIGURE 7. Local wavenumber domain analysis results for the three damage cases: (a) case 1 with the full delamination, (b) case 2 with the hidden delamination removed, (c) case 3 with half of the delamination removed. 

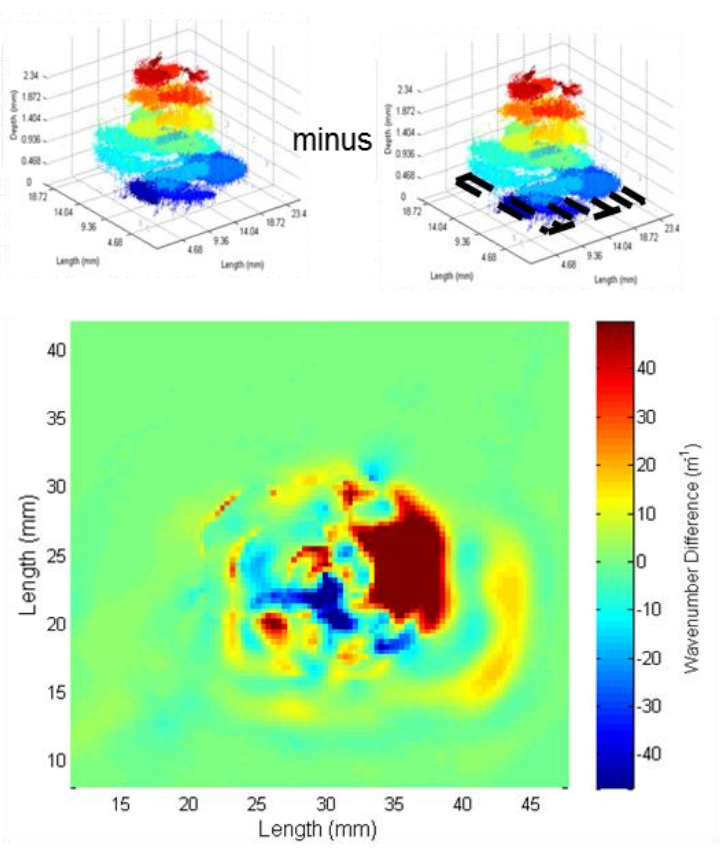

(a)
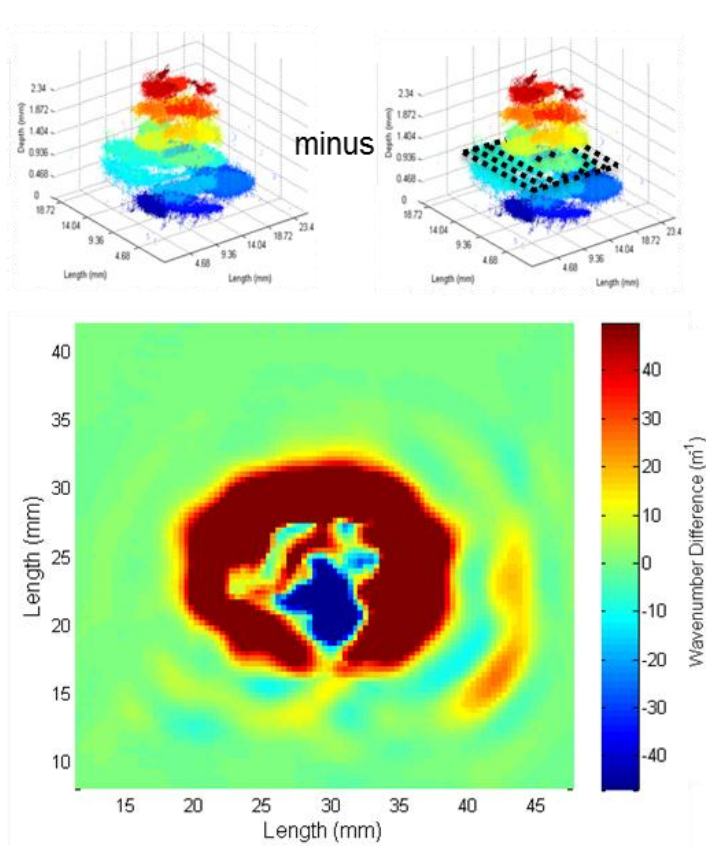

(b)

FIGURE 8. Local wavenumber differences, subtracting the wavenumber results for the cases shown above each plot: (a) result for the local wavenumber data for case 1 subtracted from the local wavenumber result for case 2, (b) local wavenumber result of case 1 subtracted from local wavenumber result for case 3. The colormap has been slightly saturated to clearly show wavenumber differences in the scattered field.
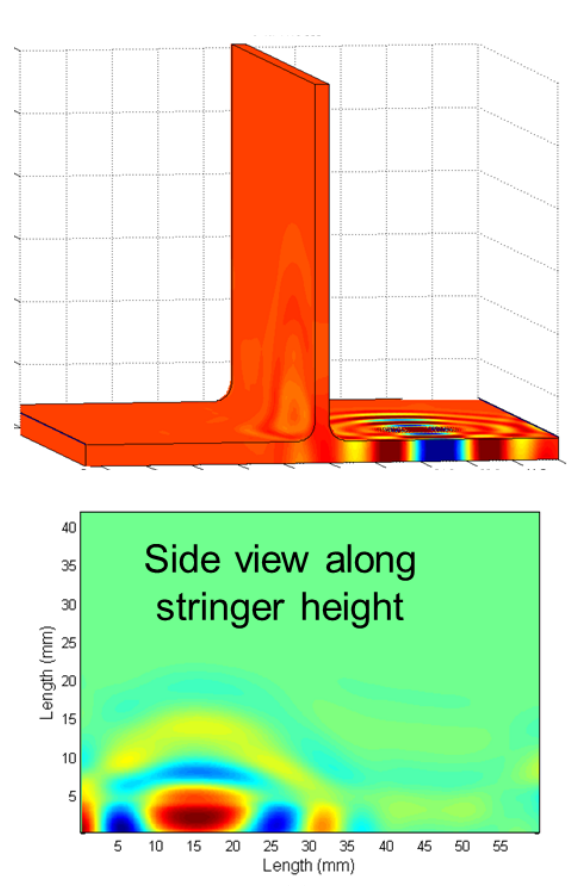

(a)
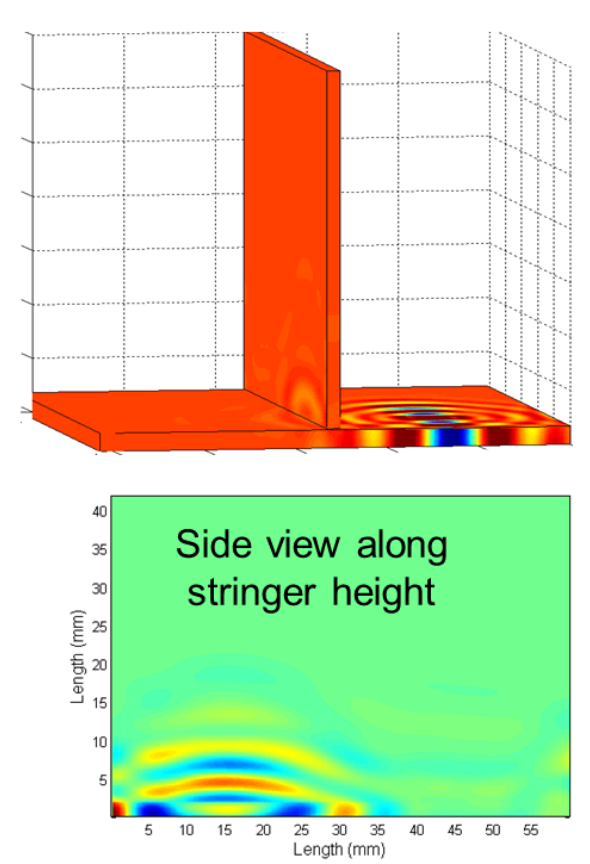

(b)

FIGURE 9. Examples of EFIT results that are a step towards simulating GW behavior in complex geometry composite components. The images show a single snapshot in time of EFIT simulation data for two cases: (a) a unidirectional composite with a curved composite T-stiffener, (b) a unidirectional composite with a simplified T-stiffener geometry. For both cases the fiber direction is into the page. Note the significant differences in GW propagation into the stiffener between the two geometries. 


\section{CONCLUSION AND FUTURE WORK}

The results presented in this paper demonstrate the type of damage characterization methods that can be investigated using 3D GW simulations that incorporate realistic composite damage. Local wavenumber domain analysis was used to investigate differences in GW behavior for three delamination damage scenarios. The three damage scenarios differed in damage severity through the composite laminate thickness, and in in-plane damage geometry. In future work, GW behavior for differing through-thickness damage scenarios will be isolated from near-surface damage geometry changes through precise removal of only the 'hidden' delamination damage. Additionally, it will be important to move beyond flat plate geometries towards GW simulation in complex geometry composite components (see figure 9). Simulations including realistic damage types and complex composite geometry components will be necessary to enable optimization and validation of NDE and SHM

techniques for future composite aerospace vehicles. Realistic simulations will also allow for predictions of inspectability of complex composite parts.

\section{ACKNOWLEDGMENTS}

The authors thank Dr. Matthew Rogge for helpful discussions and assistance in implementing local wavenumber domain analysis techniques.

\section{REFERENCES}

1. L. Yu and C. Leckey, Intell. Mater. Syst. Struct. 24, 1138-1152 (2013).

2. M. Ruzzene, Smart Mater. Struct. 16, 2116-2126 (2007).

3. M. Rogge and C. Leckey, Ultrasonics 53, 1217-1226 (2013).

4. T.E. Michaels, J.E. Michaels, M. Ruzzene, Ultrasonics 51, 452-466 (2011).

5. C. Ramadas, K. Balasubramaniam, M. Joshi, CV Krishnamurthy, Smart Mater. Struct. 19, 065009 (2010).

6. C. Leckey, M. Rogge, and F. Parker, Ultrasonics in press, DOI: 10.1016/j.ultras.2013.05.2007 (2013).

7. C. Canturri, E. Greenhalgh, S. Pinho, J. Ankersen, Compos. Part A 54, 79-87 (2013).

8. B. Pavlakovic and M. Lowe, Rev. Prog. Quant. Nondestr. 16A, 155-192 (1997). 